\title{
Fibromatosis colli spuriously presenting as a retropharyngeal mass on cervical spine radiographs
}

\author{
Katherine M. Wojcicki ${ }^{1} \cdot$ Robert H. Krieger $^{1}$ Andrew C. Berry ${ }^{2}$ (D) \\ Warren L. Reuther III $^{3}$
}

Received: 23 June 2015/ Accepted: 30 June 2015/Published online: 16 July 2015

(C) SIMI 2015

\begin{abstract}
A 6-month-old boy with a 6-month history of torticollis presented to the Emergency Department (ED) from clinic because of an outpatient cervical spine X-ray study that showed abnormal pre-vertebral soft tissue swelling in the neck, suspicious for retropharyngeal abscess (Fig. 1). The patient was referred to the ED to obtain a computed tomography (CT scan) of the neck to evaluate for abscess versus retropharyngeal mass. The patient's mother reported a 6-month history of the patient holding his head mostly to the right side with considerable right-sided neck weakness. The mother also reported that the patient had not been reaching some developmental milestones due to his inability to lift his head or sit up without support. A neurologist had recently evaluated the patient, and an electroencephalogram (EEG) was normal. The patient had a history of GERD and asthma being treated with zantac and albuterol, respectively. Birth history was unremarkable, and the patient was up to date on his immunizations. While the history was positive for neck pain to the right, it was negative for fever, drooling, difficulty breathing, neck swelling, cough, and congestion. Physical examination revealed a playful, well-developed 6-month-old male infant in no apparent distress, with no masses or swelling of the neck. The neck had a full range of motion in all directions, yet patient favored the right. The patient would not lift his
\end{abstract}

Andrew C. Berry

Aberry5555@gmail.com

1 Kansas City University of Medicine and Biosciences, Kansas City, MO, USA

2 Department of Medicine, University of South Alabama, 2451 Fillingim St, Mobile, AL 36617-2238, USA

3 Department of Radiology, West Palm Hospital, West Palm Beach, FL, USA head off the bed, roll over, or sit up on his own. He was able to drink well without difficulty. The patient's vital signs, complete blood count, and complete metabolic panel were all unremarkable. Blood cultures were negative. Neck $\mathrm{X}$-rays in neutral, flexion, and extension were obtained that showed persistent cervical pre-vertebral soft tissue thickening in all three positions. A CT scan of the neck with contrast demonstrated no retropharyngeal fluid collection or inflammatory process, and there was proper patency of the airway. Asymmetric thickening of the right sternocleidomastoid muscle, consistent with fibromatosis colli, was identified. These results, together with clinical examination, suggest the diagnosis of torticollis secondary to fibromatosis colli. The apparent pre-vertebral soft tissue thickening seen on earlier lateral X-rays was concluded to be from overlapping soft tissues secondary to torticollis.

Fibromatosis colli (FC) is a rare benign tumor of the spindle cells of the sternocleidomastoid (SCM) muscle. This lesion is characterized by diffuse or focal enlargement of the SCM muscle by fibrous tissues. It is only seen in $0.4 \%$ of infants, with $75 \%$ of these cases involving the right SCM muscle. There is a slight male predominance [1]. Although the exact etiology of FC is unknown, it is thought that FC is related to intrauterine or birth trauma resulting in ischemia and scar-like retraction of the SCM muscle. Typical examples of birth trauma leading to FC include difficult or prolonged labor, forceps assisted delivery, and breech presentation [2]. FC typically presents 2-4 weeks after birth as a nontender, palpable neck mass along the course of the SCM muscle. Decreased range of motion of the neck is common, and congenital torticollis is seen in approximately $20 \%$ of infants with FC [3].

When an infant presents with suspected congenital torticollis or a neck mass, ultrasound imaging is the preferred initial diagnostic tool due to its low cost and lack of 


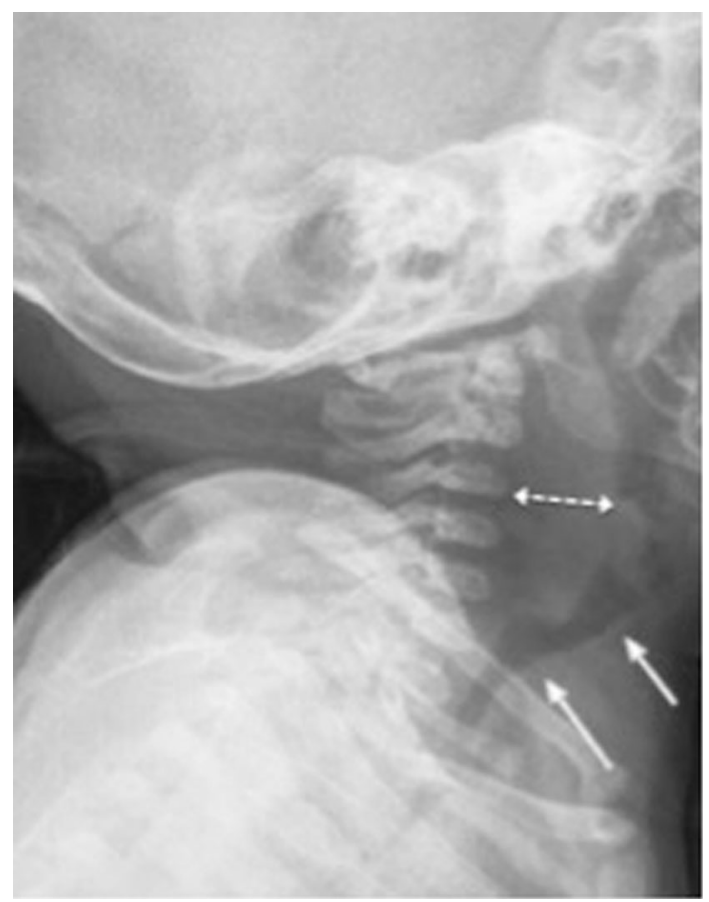

Fig. 1 A lateral X-ray of cervical spine in extension shows widening of the prevertebral soft tissues (dashed arrow) and anterior displacement of the airway (arrows). Notice the pre-vertebral soft tissues appear wider than the width of the cervical vertebral bodies at the respective level

ionizing radiation [4]. FC typically presents on ultrasound as homogenous or heterogeneous fusiform enlargement of the SCM with variable echogenicity. Plain films are usually normal, and are therefore not typically recommended for FC in the absence of other concerning symptoms. A CT scan is also not routinely recommended due to high cost and radiation exposure, but it will show iso-attenuating diffuse or focal unilateral enlargement of the SCM muscle and normal fascial planes [2]. If imaging is inconclusive, fine needle aspiration of the mass can be preformed. Cytology from the mass will reveal numerous spindle shaped fibroblasts, atrophic muscle fibers, and multinucleated regenerating muscle giant cells [5].

Fibromatosis colli is most often a self-limiting condition. In patients who present with a palpable neck mass, the mass typically increases in size for $2-3$ months, stabilizes in size, and then spontaneously resolves in 4-8 months [4]. Ninety percent of FC patients can be treated with conservative measures only, consisting of passive stretching and physiotherapy [5]. The remaining $10 \%$ of patients will demonstrate refractory FC that persists beyond 1 year of age, requiring tenotomy surgery to release the SCM muscle. Recently, Botulinum toxin type A has also been used for patients with refractory FC, further decreasing the need for surgical correction [2]. Upon discharge, our patient was instructed to sleep with the spine in a neutral position,

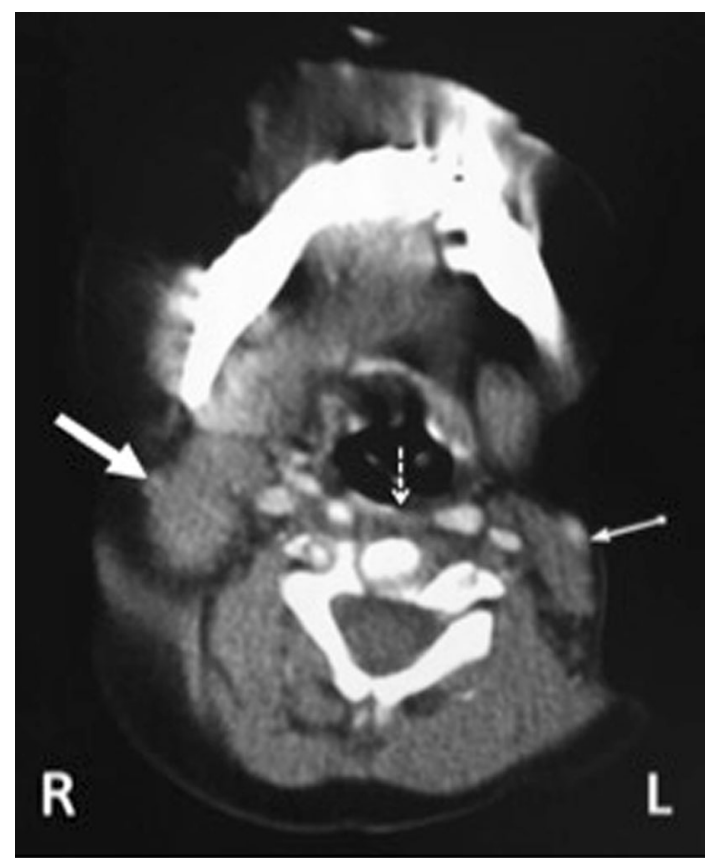

Fig. 2 A CT scan of neck with contrast shows a lack of pre-vertebral soft tissue thickening (dashed arrow), as well as proper airway patency. The right sternocleidomastoid muscle is asymmetrically enlarged (bold arrow) compared to the left (arrow)

accomplished by using a rolled up towel to keep the patient from turning his head to the right during sleep. The application of heating pads and the use of acetaminophen was also advised. With these conservative measures, our patient's fibromatosis colli was successfully treated, and within 2 months, he was able to lift his head and sit up without support. No surgical release was necessary. An EEG (electroencephalogram) 2 months after discharge was unremarkable, and the patient had no new complaints at this time.

Our patient did not present with a typical neck mass of FC, nor did he have a history of birth trauma. Our patient also had some concerning findings on physical examination, such as muscle weakness and failure to meet developmental milestones. As a result, plain films of the neck were initially ordered in order to assess osseous structures (i.e., vertebral fractures/dislocation/fusion, etc.) and spinal canal anatomy (i.e., canal diameter, instability, etc.). The lateral view radiographs showed an abnormal paravertebral soft tissue swelling in the neck, suspicious for cervical or airway trauma, or retropharyngeal mass. The prevertebral soft tissues in infants and children $<2$ years can normally measure up to $7.6 \mathrm{~mm}$ at the level of $\mathrm{C} 2$ or up to $9 \mathrm{~mm}$ at the level of $\mathrm{C} 6$ on lateral radiographs of the neck. As a generality, these tissues can approach the width of the child's cervical vertebral body, but should not exceed its width. Our patient's lateral neck radiographs (Fig. 1) demonstrate a prevertebral soft tissue width that is 
significantly greater than the width of his cervical vertebral bodies, suggesting retropharyngeal pathology. Because retropharyngeal widening on lateral X-rays can signify future airway compromise, such a finding prompts the need for further investigation. On CT images of the neck with contrast (Fig. 2), absence of prevertebral soft tissue thickening or fluid collection is seen, as well as the absence of airway narrowing. The right SCM muscle, however, shows asymmetrical enlargement compared to the left, demonstrating the cause of torticollis in this patient. No lymphadenopathy was noted, and the fascial planes surrounding the involved SCM muscle appeared normal, making an infectious etiology unlikely. The final diagnosis of this patient was fibromatosis colli, causing obliquity of the neck that was spuriously presenting as persistent prevertebral soft tissue widening on a lateral neck X-ray study. This patient's condition was likely due to intrauterine injury such as abnormal fetal head position, for the patient had no history of trauma at birth or during the neonatal period [1].

This case demonstrates the unusual presentation of fibromatosis colli as a retropharyngeal mass on lateral neck radiographs. Although conventional radiographs are not traditionally recommended in the work up of fibromatosis colli, this case provides evidence that torticollis and overlying soft tissue structures can cause an apparent false widening of prevertebral soft tissue on lateral neck X-rays. In addition, this case demonstrates that fibromatosis colli can be an underlying cause of torticollis, even in the absence of a classic neck mass or swelling.

\section{Compliance with ethical standards}

Conflict of interest The authors declare that they have no conflict of interest.

Statement of human and animal rights All procedures performed in studies involving human participants were in accordance with the ethical standards of the institutional and/or national research committee and with the 1964 Helsinki declaration and its later amendments or comparable ethical standards. This article does not contain any studies with animals performed by any of the authors.

Informed consent Informed consent was obtained from all individual participants included in the study.

\section{References}

1. Adamoli P, Pavone P, Falsaperla R et al (2014) Rapid spontaneous resolution of fibromatosis colli in a 3-week-old girl. Case Rep Otolaryngol 2014:264940

2. Khalid S, Zaheer S, Wahab S, Siddiqui MA, Redhu N, Yusuf F (2012) Fibromatosis colli: a case report. Oman Med J 27(6)

3. Flanagan SM, Yeom KW, Barnes PD, Newman B (2014) Fibromatosis colli. In: Daldrup-Link HE, Newman B (eds) Pearls and pitfalls in pediatric imaging-variants and other difficult diagnoses. Cambridge University Press, Cambridge

4. Haque S, Shafi BBB, Kaleem M (2012) Imaging of torticollis in children. Radiogr Pediatr Imaging 32(2):557-571

5. Khan S, Jetley S, Kairajpuri Z, Husain M (2014) Fibromatosis colli-a rare cytological diagnosis in infantile neck swellings. J Clin Diagn Res 8(11):FD08-FD09 